\title{
TRANSPLANT CANDIDATE'S CLINICAL STATUS RATHER THAN RIGHT VENTRICULAR FUNCTION DEFINES NEED FOR UNIVENTRICULAR VERSUS BIVENTRICULAR SUPPORT
}

Robert L. Kormos, $\mathrm{MD}^{\mathrm{a}}$

Thomas A. Gasior, $\mathrm{MD}^{\mathrm{b} \S}$

Akihiko Kawai, $\mathrm{MD}^{\mathrm{a}}$

Si M. Pham, MD ${ }^{\text {as }}$

Srinivas Murali, $\mathrm{MD}^{\mathrm{c} \&}$

Brack G. Hattler, MD ${ }^{\text {s }}$

Bartley P. Griffith, MD
We have studied our experience since 1988 with 31 patients who required a mechanical circulatory bridge to transplantation and also had biventricular failure (mean right ventricular ejection fraction $11.8 \%$ ) to better define the need for biventricular or total artificial heart support versus univentricular support. Clinical factors including preoperative inotropic need, fever without detectable infection, diffuse radiographic pulmonary edema, postoperative blood transfusion, and right ventricular wall thickness were compared with hemodynamic parameters including cardiac index, right ventricular ejection fraction, central venous pressure, mean pulmonary arterial pressure, and total pulmonary resistance for ability to predict need for mechanical or high-dose inotropic support for the right ventricle. Patients were grouped according to need for right ventricular support after left ventricular-assist device implantation: none (group $A, 14$ ) inotropic drugs (group $B_{1}, 7$ ), and right ventricle mechanical support (group $B_{2}, 10$ ). There were no differences in preimplantation hemodynamic variables. Groups $B_{1}$ and $B_{2}$ had significantly lower mixed venous oxygen saturation $(39.2 \%$ ys $52.5 \%$ in group $A ; p<0.001)$, greater level of inotropic need $(p<0.02)$, greater impairment of mental status, and lower ratio of right ventricular ejection fraction to inotropic need $(0.37$ vs 0.56 for group $A ; p<0.02$ ) before left ventricular-assist device implantation. A significant discriminator between groups $B_{1}$ and $B_{2}$ was the presence of a fever without infection within 10 days of left ventricular-assist device implantation (43\% in group $B_{1}$ vs $70 \%$ in group $B_{2}$ ). Group $B_{2}$ had more patients with preimplantation pulmonary edema seen on chest radiography and a greater requirement for postoperative blood transfusion (5 units of cells in group $B_{1}$ vs 14.8 units in group $B_{2}$. Right ventricular wall thickness at left ventricularassist device explantation was $0.83 \mathrm{~cm}$ in group $B_{2}$ vs $0.44 \mathrm{~cm}$ in group $B_{1}(p<$ 0.05 ). Transplantation rates after bridging were $100 \%$ in group $A, 71 \%$ in group $B_{1}$, and $40 \%$ in group $B_{2}$. Clinical factors that reflect preimplantation degree of illness and perioperative factors that result in impairment of pulmonary blood flow or reduced perfusion of the right ventricle after left ventricular-assist device implantation are now considered to be more predictive of the need for additional right ventricular support than preimplantation measures of right ventricular function or hemodynamic variables. (J THORAC CARDIOvaSc Surg 1996;111:773-83)
From the Department of Surgery, University of Pittsburgh School of Medicine and Veterans Administration Medical Center, ${ }^{a}$ and the Departments of Anesthesiology ${ }^{\mathrm{b}}$ and Cardiology, University of Pittsburgh School of Medicine, Pittsburgh, Pa.

Read at the Seventy-fifth Annual Meeting of The American Association for Thoracic Surgery, Boston, Mass., April 23-26, 1995.

Received for publication April 26, 1995; accepted for publication Oct. 26, 1995; revisions requested Sept. 5, 1995; revisions received Oct. 24, 1995.

Address for reprints: Robert L. Kormos, MD, University of Pittsburgh, Presbyterian University Hospital, Cardiothoracic Surgery Division, 200 Lothrop St.-Suite C700, Pittsburgh, PA 15213. linical trials with left ventricular-assist devices $\mathcal{U}$ (LVADs) as bridges to cardiac transplantation continue to suggest a potential role for such devices as an alternative to cardiac transplantation. ${ }^{1-3} \mathrm{Be}$ fore applicability of such devices in this role can be assumed, however, a more complete understanding of the target population must be demonstrated. Perhaps the least answered question in this respect

${ }^{\S}$ By invitation.

$12 / 6 / 70331$ 
relates to the selection of patients on the basis of adequacy of right ventricular performance. This is a particularly difficult dilemma because no preimplantation criteria as yet exist for predicting the postimplantation performance of the right ventricle in hearts supported by LVADs. ${ }^{4,5}$

Initial reports from our institution were optimistic in the hope that most patients with end-stage heart failure could survive with univentricular support alone. $^{6,7}$ Unfortunately there is a $10 \%$ to $30 \%$ reported incidence of right ventricular failure necessitating right ventricular mechanical support in addition to the LVAD, with a mortality rate in this setting of $30 \%$ to $50 \%{ }^{8}$ Some reports have acknowledged that right and left ventricular size, ventricular filling pressures, ventricular ejection fractions, and standard hemodynamic parameters are incapable of predicting which patients are at risk for inadequate right ventricular performance to support adequate LVAD cardiac output, ${ }^{3}$ whereas others have suggested that a right atrial pressure greater than 20 $\mathrm{mm} \mathrm{Hg}$ predicts the need for biventricular assistance. ${ }^{9}$ Farrar and coworkers ${ }^{10}$ recently reported results with 105 left and 195 biventricular Thoratec ventricular-assist devices (Thoratec Laboratories Corp., Berkeley, Calif.) showing that preassist cardiac indexes were lower among persons requiring biventricular support and that preimplantation serum creatinine levels were higher. ${ }^{10}$ In addition, they showed that a greater percentage of patients who underwent biventricular support were receiving mechanical ventilation before support, and more had implantation in an emergency setting than among patients who had the LVAD alone. From our early observations, we suspected that clinical events surrounding LVAD implantation may be more predictive of the success of univentricular support than any preoperative functional or hemodynamic measurement. This report documents our review of hemodynamic, physiologic, and clinical data collected for patients receiving the Novacor left ventricular-assist system (LVAS; Novacor Medical Division, Baxter Healthcare Corp., Oakland, Calif.) as a bridge to cardiac transplantation. Our goal was to identify variables that may be predictive of subsequent right ventricular failure.

\section{Materials and methods}

Patient selection. Between May 1988 and March 1994, 43 candidates for orthotopic transplantation could not be adequately supported hemodynamically by multiple inotropic agents and an intraaortic balloon pump (IABP) before cardiac transplantation and required additional support from the Novacor N-100 LVAS as a bridge to transplantation. Thirty-three received the console-based system and 10 received the wearable, portable device. Patient selection criteria, description of the device, implantation technique, management, and indications for transplantation were published previously. ${ }^{7}$ Detailed and complete prospective data were available for the last 40 of 43 consecutive patients ( $93 \%$ ); these data form the basis for this report. There were 37 male and three female patients, ranging in age from 15 to 63 years (mean 43.6 years). Thirteen patients had ischemic cardiomyopathy and 27 had idiopathic dilated cardiomyopathy. The body surface area of the patients ranged from 1.5 to $2.3 \mathrm{~m}^{2}$ (mean $1.9 \mathrm{~m}^{2}$ ). All patients required two or more inotropic agents, and all but nine required an LABP before LVAS insertion. An IABP was not inserted in the presence of severe supraventricular or ventricular arrhythmias, which made IABP timing nearly impossible. Average durations of inotropic support and IABP support were 24 days (range 2 to 71 days) and 11 (range 1 to 43 days), respectively.

All consent forms, implantation techniques, and protocols for hemodynamic and biomechanical evaluation were examined and approved by the University of Pittsburgh Institutional Review Board. An investigational device exemption was granted by the Food and Drug Administration for the original console-based system in July 1987 and for the wearable portable system in March 1993. Informed consent was obtained from all patients or next of kin. All patients who received support were candidates for orthotopic transplantation and underwent such transplantation as soon as a donor organ could be found and it was clear that there were no contraindications to transplantation.

Hemodynamic data. Immediately before induction of anesthesia for LVAS implantation, a rapid-response thermodilution catheter (REF catheter; Bentley Division; Baxter Healthcare Corp., Irvine, Calif.) was placed into the pulmonary artery through the right or left internal jugular vein. A baseline hemodynamic profile consisting of mean arterial pressure, heart rate, mean pulmonary artery pressure, and pulmonary capillary wedge pressure was obtained. The cardiac output computer provided right ventricular ejection fraction (RVEF), right ventricular end-diastolic and end-systolic volumes, right ventricular stroke volume, right ventricular stroke work index, and cardiac output, from which cardiac index was calculated. The RVEF measurement was confirmed by calculating right ventricular fractional area change obtained by transesophageal echocardiography with epicardial two-dimensional images recorded with a Hewlett Packard Sonos 1500 Ultra Sound System with automated border-detection capabilities (model 77035A; Hewlett Packard Co., Medical Products Group, Andover, Mass.). Total pulmonary resistance was calculated by multiplying the mean pulmonary artery pressure by 80 and dividing the result by cardiac output. These measurements were made prospectively before and after induction of anesthesia and at 20 , 40 , and 60 minutes after the LVAS was started. They were repeated at the time of sternal closure and at 8,24 , and 48 hours after startup. At least three separate measurements 
were made and averaged for each data record. Transesophageal echocardiography was also employed intraoperatively to assess proper implantation technique.

Clinical data. Clinical data was recorded prospectively from patient charts including intensive care unit and anesthesia records. An attempt was made to standardize the amount of inotropic support needed for right ventricular support before and after implantation of the LVAS. The inotropic levels were expressed as inotropic equivalents (IEs), with $1 \mu \mathrm{g} \cdot \mathrm{kg}^{-1} \cdot \mathrm{min}^{-1}$ dopamine, dobutamine, or amrinone equal to $1 \mathrm{IE}, 1 \mu \mathrm{g} \cdot \mathrm{kg}^{-1} \cdot \mathrm{min}^{-1}$ milrinone equal to $15 \mathrm{IE}$, and $0.1 \mu \mathrm{g} \cdot \mathrm{kg}^{-1} \cdot \mathrm{min}^{-1}$ epinephrine or norepinephrine representing $10 \mathrm{IE}$. In an effort to normalize the IEs needed to support the right ventricle before and after LVAS implantation, RVEF (obtained by the thermodilution technique) was divided by the IEs of support used at each point of measurement (RVEF/IE ratio). Patients were classified according to pre-LVAS RVEF (greater or less than $20 \%$ ). In addition, they were subcategorized according to the need for large amounts of inotropic or mechanical support for the right ventricle after LVAS implantation. Right ventricular failure after LVAS implantation was defined as the need for a right ventricular-assist device, despite inotropic support, to maintain the LVAS cardiac index at greater than 2.0 $\mathrm{L} \cdot \min ^{-1} \cdot \mathrm{m}^{-2}$. In all cases, an attempt was made to manage the right ventricle initially with inotropic agents alone; however, a right ventricular-assist device in the form of a centrifugal pump (Medtronic Bio-Medicus, Eden Prairie, Minn.) was inserted between 8 and 24 hours after the initial implant of the LVAS if needed. Mixed venous oxygen saturation was obtained from the pulmonary artery catheter in the intensive care unit and represented the last measurement before the decision for LVAS implantation or just before transfer to the operating room. Preimplantation mental status was expressed by a score of 0 to $3(0$, normal; 1 , mildly confused or drowsy but appropriately oriented; 2 , drowsy but responding to verbal stimuli; 3 , drowsy and responding only to pain, or intubated). The presence or absence of pulmonary edema or diffuse interstitial pulmonary infiltrate noted on the most recent preimplantation chest radiograph was recorded retrospectively from a review by an independent radiographer. The instances of elevation of body temperature to greater than $38.0^{\circ} \mathrm{C}$ before implantation were noted, and in the presence of an identifiable source of infection appropriate intravenous antibiotic therapy was started and completed before device implantation. No devices were implanted in the presence of a known infection that had not resolved, and a period of at least 10 days of normothermia was required. Cases of fever alone were not considered a contraindication to LVAS implantation when no identifiable infectious source could be identified and repeated blood cultures were sterile. For purposes of this study, only occurrences of fever without septicemia were recorded. The blood products used perioperatively for LVAS implantation were recorded and included units of platelets, fresh-frozen plasma, packed erythrocytes, and cryoprecipitate. Recorded amounts included only those amounts transfused before any additional surgery for a right ventricular-assist device. Pathologic examination of the explanted heart was made either during autopsy at patient death or after orthotopic transplantation. Right and left ventricular wall thickness and any right ventricular hypertrophy and fibrosis were noted.

Data analysis. Patients were classified into groups on the basis of the pre-LVAS RVEF. Only those patients with RVEF less than or equal to $20 \%$ were statistically analyzed with respect to variables that could predict subsequent right ventricular failure and the need for right ventricular mechanical assistance. Further classification was then made on the basis of ease of clinical management of the right ventricle after LVAS implantation: group A, short-term inotropic support (less inotropic support at 8 hours after implantation than before implantation); group $\mathbf{B}_{1}$, high-dose inotropic support (greater dosage at 8 hours than before implantation; and group $B_{2}$, ultimately requiring mechanical right ventricular support. Actuarial survival was calculated with the Kaplan-Meier method. Previous work had shown that a critical point in the clinical course of these patients occurred at the point of sternal closure, with no significant changes occurring from then until 8 hours after LVAS implantation or until a right ventricular-assist device was needed. For that reason, hemodynamics at sternal closure were taken as the most representative post-LVAS values. Differences in means were tested with paired and unpaired $t$ tests for normal data, and nonparametric tests (Mann-Whitney) were used in other cases. Comparisons were made between group $\mathrm{A}$ and $\mathrm{B}$ patients and between the subgroups $\mathrm{B}_{1}$ and $\mathrm{B}_{2}$. The $\chi^{2}$ test was used to test the significance of differences in proportions between groups, and the Fisher's Exact Test was used for small sample sizes. A $p$ value less than 0.05 was taken as the level of significance. Statistics were performed with STATISTICA for Windows (StatSoft, Inc., Tulsa, Okla.).

\section{Results}

Overall results. Of 40 patients who underwent LVAS implantation, 31 (78\%) survived to transplantation and 27 were discharged $(68 \%)$. Causes of death during device support included persistent multiorgan failure (five), cerebral hemorrhage (two), infection (one), and technical causes (one). Deaths after transplantation were from infection (fungal, one; toxoplasmosis, one), acute allograft rejection (one), and perforated gastric ulcer (one). Actuarial survival after cardiac transplantation after LVAS support was $90 \%$ at 2 years and $73 \%$ at 4 years.

Patient outcome according to preimplantation RVEF is shown in Fig. 1. Nine patients had preimplant RVEFs greater than $20 \%$ (mean $33.2 \% \pm$ $7.5 \%$ ). No right ventricular dysfunction was seen as a result of LVAS implantation in any patient in this group, and inotropic agent usage was reduced within 24 hours in all cases and was discontinued within an average of 8 days. Seven of these patients $(78 \%)$ underwent successful heart transplantation and 


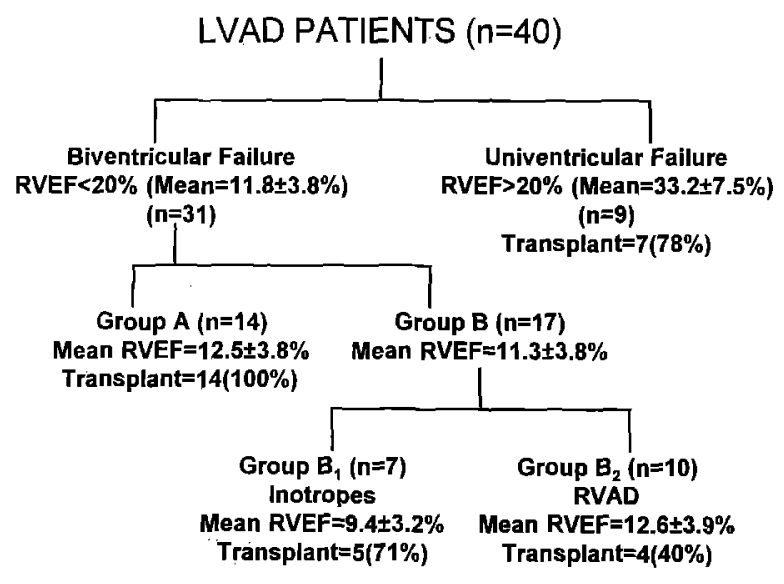

Fig. 1. Summary of Novacor LVAS recipient groups according to preimplantation RVEF and post-LVAS clinical course. Transplantation outcomes are shown. RVAD, Right ventricular-assist device.

were discharged; one died while on support of septicemia from a pocket infection resulting from obesity when the device could not be placed below the rectus muscle, and another died while on support of myocardial infarction. The remaining 31 patients all had right ventricular dysfunction before LVAS insertion (mean RVEF $11.8 \% \pm 3.8 \%$ ) and form the core group for analysis of factors predicting the need for right ventricle support. This group was further classified according to the ease of management of the right ventricle after LVAS implantation. Group A $(N=14)$ required short-term (mean $<7$ days) of inotropic support. All underwent heart transplantation and were successfully discharged from hospital. Group B $(N=17)$ required either high-dose inotropic support (group $\mathrm{B}_{1}, N=$ 7 ) or the addition of a right ventricular-assist device (group $\mathrm{B}_{2}, N=10$ ). Five patients $\left(71 \%\right.$ ) in group $\mathrm{B}_{1}$ underwent successful transplantation; two others died of cerebrovascular complications. Only four patients in group $\mathrm{B}_{2}(40 \%)$ survived to undergo transplantation; the remainder died of progressive multiorgan failure during LVAS support.

Hemodynamic results. A summary of hemodynamic measurements in group $\mathrm{A}$ and $\mathrm{B}$ patients before and after LVAS implantation (at the time of sternal closure) are summarized in Fig. 2. Significant reductions were seen after LVAS implantation in pulmonary capillary wedge pressure, pulmonary artery mean pressure $(36.0$ vs $24.8 \mathrm{~mm} \mathrm{Hg} ; p<$ 0.0001 ), and total pulmonary resistance for both groups. The total pulmonary resistance in group $\mathrm{A}$
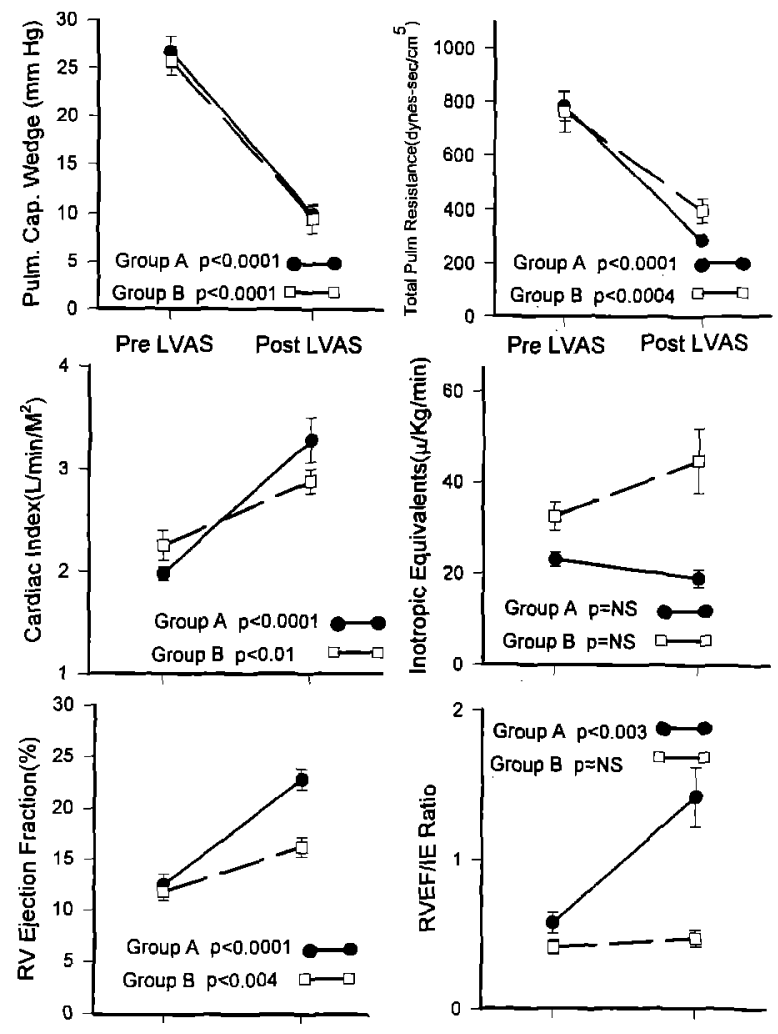

Fig. 2. Hemodynamic changes before (PreLVAS) and after (PostLVAS) Novacor implantation. NS, Not significant.

was significantly lower $(p<0.04)$ than in group B after LVAS implantation. Accompanying these changes were significant elevations in cardiac index and RVEF. The cardiac index was similar in both groups after LVAS implantation; however, RVEF was significantly higher in group A after LVAS implantation ( $22.8 \%$ vs $16.2 \% ; p<0.0001)$. Although inotropic support required at sternal closure did not change significantly from pre-LVAS levels in either group, the degree of inotropic support was significantly higher in group B patients after LVAS (44.7 vs 18.8 IE; $p<0.004$ ). There was a significant rise in the RVEF/IE ratio after LVAS implantation in group A but not in group B, and this ratio was significantly higher after LVAS implantation in group A (1.4) than in group B $(0.5 ; p<0.0001)$. There were no significant changes in central venous pressure after LVAS implantation in either group, nor were there differences between groups after LVAS insertion. No significant differences could be seen in hemodynamic response to LVAS between group $B_{1}$ and $B_{2}$ patients, and there were also no 
differences in any of the immediate post-LVAS hemodynamic measurements between these groups. There were no significant differences between group $\mathrm{A}$ and $\mathrm{B}$ patients with respect to right-sided or left-sided filling pressures, mean pulmonary artery pressure, heart rate, cardiac index, total pulmonary resistance, or right ventricular ejection fraction before LVAS implantation (Table I). Likewise, no differences were noted in the right ventricular enddiastolic or end-systolic volumes between these groups of patients before LVAS implantation. Patients in group B had a greater level of inotropic support before LVAS than did those in group A $(p<0.02)$, and in fact the level of inotropic support was higher in group $\mathrm{B}_{2}$ than in group $\mathrm{B}_{1}(p<0.05)$. Finally, the RVEF/IE ratio was significantly lower in group B patients before LVAS (0.37) than in group A patients $(0.56 ; p<0.02)$.

Clinical results. A summary of pre-LVAS clinical variables for the three groups is shown in Table II. No significant differences were seen between groups $A$ and $B_{1}$ or between groups $B_{1}$ and $B_{2}$ with respect to age, body surface area, gender, etiology of cardiomyopathy, or duration of support with inotropic agents or IABP before LVAS. Evidence of pulmonary edema on a chest radiograph was seen just before LVAS implantation in 53\% of group B and $14 \%$ of group A patients $(p<0.03)$. There was also a greater prevalence of this finding among patients who ultimately needed a right ventricular-assist device $(60 \%)$ than among those who did not $(43 \%)$; however this difference did not reach statistical significance. A significantly higher proportion of group B patients $(41 \%)$ had a greater degree of mental impairment (mental score greater than or equal to 2) than among group A patients (7\%) just before LVAS implantation $(p<0.04)$; however, groups $B_{1}$ and $B_{2}$ did not differ in this regard. The RVEF/IE ratio at the time of sternal closure appeared to be related to the pre-LVAS mental status (Fig. 3), with a lower ratio seen in patients with a mental status score of 2 or less $(p<0.004)$. The prevalence of fever (in the absence of infection) within 10 days before LVAS implantation was higher among group $B$ than among group $A$ patients (47\% vs $14 \%$ ) and was also higher among group $\mathrm{B}_{2}$ $(70 \%)$ than among group $\mathrm{B}_{1}(14 \% ; p<0.02)$. In patients with fever, the average time from last fever to LVAS implantation was significantly shorter among group $B_{2}$ patients ( 6 days) than among group $\mathrm{B}_{1}$ patients $(18$ days; $p<0.02)$. Preimplantation hematologic, biochemical, and metabolic values are

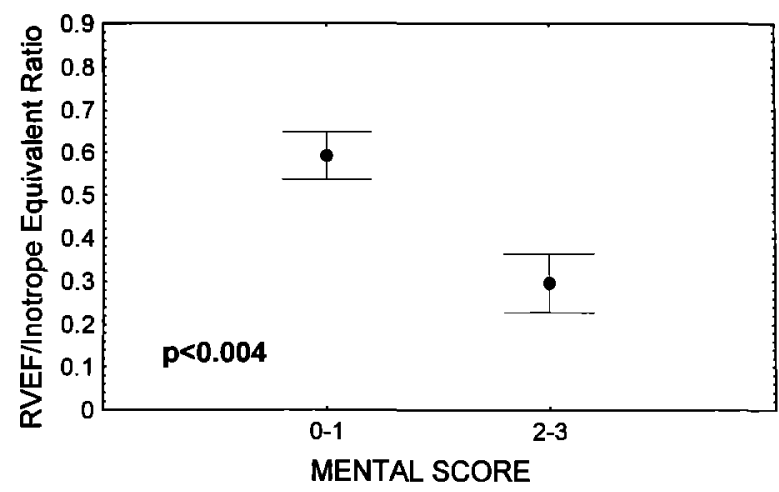

Fig. 3. RVEF/IE ratio at sternal closure in group B patients. Averages are shown for patients with minimal or no mental dysfunction before LVAS implantation (score 0 or 1) and for patients with moderate to severe mental dysfunction (score 2 or 3 ).

shown in Table III. The peak creatinine levels were significantly higher in group $\mathrm{B}$ than in group A patients $(2.1 \pm 0.7$ vs $1.5 \pm 0.6 \mathrm{mg} / \mathrm{dl})$ but did not differ between groups $B_{1}$ and $B_{2}$. Although the peak bilirubin level did not differ significantly between groups $\mathrm{A}$ and $\mathrm{B}$, there was a trend toward higher preimplantation bilirubin levels in group $B_{2}$ than in group $B_{1}$ patients $(3.3 \pm 2.9$ vs $2.2 \pm 0.7 \mathrm{mg} / \mathrm{dl})$. There were no significant differences in preimplantation hemoglobin levels, leukocyte counts, platelet counts, prothrombin times, and partial thromboplastin times between the groups of patients (A vs B; $B_{1}$ vs $\left.B_{2}\right)$. Finally, mixed venous oxygen saturation immediately before LVAS implantation was significantly lower in group B than in patients in group A (39.2\% vs $52.5 \% ; p<0.001)$.

One major complication seen after LVAS implantation was perioperative bleeding. Forty-three percent of group A and 88\% of group B patients had perioperative bleeding that required blood product transfusion $(p<0.02)$. In $80 \%$ of the cases, this bleeding was caused by coagulopathy or diffuse bleeding from the outflow conduit and was not related to anastomotic or surgical technique. Although the difference was not statistically different, $100 \%$ of group $B_{2}$ patients had perioperative bleeding complications, compared with $71 \%$ of the group $\mathrm{B}_{1}$ patients. Blood product usage in the perioperative phase is shown in Table IV. Significantly greater amounts of erythrocytes $(p<0.01)$, fresh-frozen plasma $(p<0.05)$, platelets $(p<0.01)$, and cryoprecipitate $(p<0.05)$ were transfused into group B patients than into group $A$ patients, and the trend 
Table I. Pre-LVAS hemodynamic variables for $L V A S$ recipients according to clinical group

\begin{tabular}{|c|c|c|c|c|c|c|}
\hline & Group $A$ & Group B & $\begin{array}{c}p \\
\text { (group } A \text { vs group B) }\end{array}$ & Group $B_{1}$ & Group $B_{2}$ & $\begin{array}{c}p \\
\text { (group } B_{1} \text { is group } B_{2} \text { ) }\end{array}$ \\
\hline $\begin{array}{l}\text { Pulmonary capillary wedge pres- } \\
\text { sure (mm } \mathrm{Hg} \text { ) }\end{array}$ & $26.4 \pm 5.9$ & $25.7 \pm 6.1$ & NS & $26.7 \pm 7.93$ & $25.1 \pm 4.7$ & NS \\
\hline $\begin{array}{l}\text { Pulmonary artery mean pressure } \\
\text { (mm Hg) }\end{array}$ & $36.8 \pm 8.1$ & $37.6 \pm 8.4$ & NS & $9.4 \pm 12$ & $36.3 \pm 5.1$ & NS \\
\hline Central venous pressure $(\mathrm{mm} \mathrm{Hg})$ & $16.6 \pm 7.5$ & $16.2 \pm 4.9$ & NS & $18.4 \pm 5$ & $14.8 \pm 4.5$ & NS \\
\hline Heart rate (beats/min) & $100 \pm 16$ & $110 \pm 21$ & NS & $117 \pm 21$ & $105 \pm 21$ & NS \\
\hline Cardiac index $\left(\mathrm{L} \cdot \min ^{-1} \cdot \mathrm{m}^{-2}\right)$ & $1.9 \pm 0.2$ & $2.2 \pm 0.6$ & NS & $2.0 \pm 0.4$ & $2.4 \pm 0.7$ & NS \\
\hline $\begin{array}{l}\text { Total Pulmonary Resistance } \\
\left(\text { dynes } \cdot \mathrm{sec} \cdot \mathrm{cm}^{-5} \text { ) }\right.\end{array}$ & $782 \pm 201$ & $745 \pm 340$ & NS & $897 \pm 391$ & $639 \pm 272$ & NS \\
\hline $\operatorname{RVEF}(\%)$ & $12.5 \pm 3.8$ & $11.3 \pm 3.9$ & NS & $9.4 \pm 3.2$ & $12.6 \pm 3.9$ & NS \\
\hline $\mathrm{IE}\left(\mu \mathrm{g} \cdot \mathrm{kg}^{-1} \cdot \min ^{-1}\right)$ & $23.6 \pm 5.7$ & $32.7 \pm 12.6$ & $<0.02$ & $26.0 \pm 5.1$ & $37.3 \pm 14.3$ & $<0.05$ \\
\hline RVEF/IE ratio & $0.56 \pm 0.27$ & $0.37 \pm 0.14$ & $<0.02$ & $0.36 \pm 0.08$ & $0.38 \pm 0.18$ & NS \\
\hline $\begin{array}{l}\text { Right ventricular end-diastolic } \\
\text { volume (ml) }\end{array}$ & $334 \pm 69$ & $372 \pm 128$ & NS & $333 \pm 86$ & $399 \pm 149$ & NS \\
\hline $\begin{array}{l}\text { Right ventricular end-diastolic } \\
\text { volume index }(\mathrm{ml})\end{array}$ & $175 \pm 34$ & $197 \pm 63$ & NS & $183 \pm 53$ & $206 \pm 70$ & NS \\
\hline $\begin{array}{l}\text { Right ventricular end-systolic vol- } \\
\text { ume (ml) }\end{array}$ & $295 \pm 74$ & $330 \pm 121$ & NS & $298 \pm 841$ & $352 \pm 141$ & NS \\
\hline $\begin{array}{l}\text { Right ventricular end-systolic vol- } \\
\text { ume index (ml) }\end{array}$ & $154 \pm 36$ & $174 \pm 59$ & NS & $163 \pm 52$ & $181 \pm 66$ & NS \\
\hline
\end{tabular}

NS, Not significant.

Table II. Clinical variables for $L V A S$ recipients according to clinical group

\begin{tabular}{|c|c|c|c|c|c|c|}
\hline & Group $A$ & Group B & $\begin{array}{c}p \\
(A \vee s B)\end{array}$ & Group $B_{I}$ & Group $B_{2}$ & $\begin{array}{c}p \\
\left(B_{1} v s B_{2}\right)\end{array}$ \\
\hline$N$ & 14 & 17 & & 7 & 10 & \\
\hline Age (yr) & $45.5 \pm 12.4$ & $41.5 \pm 13.0$ & NS & $40.3 \pm 9.8$ & $42.2 \pm 15.4$ & NS \\
\hline Body surface area $\left(\mathrm{m}^{2}\right)$ & $1.9 \pm 0.2$ & $1.9 \pm 0.2$ & NS & $1.8 \pm 0.1$ & $1.9 \pm 0.2$ & NS \\
\hline Percent female & 0 & 18 & NS & 28 & 10 & NS \\
\hline Percent with idiopathic CM & 64 & 82 & NS & 71 & 90 & NS \\
\hline Percent with ischemic CM & 36 & 18 & NS & 29 & 10 & NS \\
\hline Percent with mental score $\geq 2$ & 7 & 41 & $<0.04$ & 40 & 43 & NS \\
\hline $\begin{array}{l}\text { Percent with pulmonary edema before } \\
\text { implantation }\end{array}$ & 14 & 53 & $<0.03$ & 43 & 60 & NS \\
\hline Febrile days before implantation & $27.0 \pm 41$ & $9.6 \pm 8.1$ & NS & $18 \pm 7.2$ & $5.6 \pm 5.1$ & $<0.02$ \\
\hline $\begin{array}{l}\text { Percent with fever } \leq 10 \text { days before im- } \\
\text { plantation }\end{array}$ & 14 & 47 & NS & 14 & 70 & $<0.02$ \\
\hline $\begin{array}{l}\text { Preimplantation days of inotropic sup- } \\
\text { port }\end{array}$ & $22.6 \pm 13.3$ & $26.8 \pm 13.4$ & NS & $22.6 \pm 19$ & $29.7 \pm 18$ & NS \\
\hline Preimplantation days of IABP support & $8.1 \pm 9.2$ & $10.9 \pm 11.2$ & NS & $15 \pm 14$ & $7.8 \pm 8.0$ & NS \\
\hline
\end{tabular}

$\overrightarrow{N S}$, Not significant.

continued, with more of these blood products being required in group $B_{2}$ patients than in group $B_{1}$ patients. Significant infections necessitating antibiotics occurred in $14 \%$ of group A patients and $29 \%$ of group B patients ( $p$ not significant). In both groups of patients the pre-LVAS IABP support time was longer (mean $22.7 \pm 14$ days) in those who acquired an infection after LVAS implantation than in those who did not (mean $7.6 \pm 8$ days; $p<0.002$ ). Significant neurologic events occurred in $28 \%$ of group A and $12 \%$ of group B patients during LVAS support. Although there were no significant differences noted between group A and group B patients with respect to weight of the heart at explantation (471 vs $515 \mathrm{gm}$ ), right ventricular wall thickness ( 0.64 vs $0.67 \mathrm{~cm})$, left ventricular thickness (1.6 vs $1.6 \mathrm{~cm}$ ), and right ventricular myocyte hypertrophy, there was a significant difference in right ventricular wall thickness between group $B_{2}(0.83 \mathrm{~cm})$ and group $\mathrm{B}_{1}(0.44 \mathrm{~cm})$ patients $(p<0.05)$. 
Table III. Biochemical, hematologic, metabolic, and coagulation parameters for LVAS recipients according to clinical group

\begin{tabular}{|c|c|c|c|c|c|c|}
\hline & Group A & Group B & $\begin{array}{c}p \\
(A \vee s B) \\
\end{array}$ & Group $B_{1}$ & Group $B_{2}$ & $\begin{array}{c}p \\
\left(B_{1} v S B_{2}\right) \\
\end{array}$ \\
\hline Peak creatinine before implantation $(\mathrm{mg} / \mathrm{dl})$ & $1.5 \pm 0.6$ & $2.1 \pm 0.7$ & $<0.02$ & $2.1 \pm 0.6$ & $2.2 \pm 0.8$ & NS \\
\hline Peak bilirubin before implantation $(\mathrm{mg} / \mathrm{dl})$ & $3.9 \pm 6.6$ & $2.9 \pm 2.3$ & NS & $2.2 \pm 0.7$ & $3.3 \pm 2.9$ & NS \\
\hline Preimplantation hemoglobin $(\mathrm{gm} / \mathrm{dl})$ & $11.0 \pm 2.1$ & $10.9 \pm 1.4$ & NS & $10.8 \pm 1.7$ & $10.9 \pm 1.2$ & NS \\
\hline Preimplantation leukocyte count (cells $\times 10^{3} / \mathrm{mm}^{3}$ ) & $10.9 \pm 5.3$ & $10.9 \pm 3.8$ & NS & $9.3 \pm 3.7$ & $12.0 \pm 3.6$ & NS \\
\hline Preimplantation platelet count (cells $\times 10^{3} / \mathrm{mm}^{3}$ ) & $208 \pm 91$ & $156 \pm 82$ & NS & $141 \pm 39$ & $167 \pm 103$ & NS \\
\hline Mixed venous oxygen saturation (\%) & $52.5 \pm 7.4$ & $39.2 \pm 10.4$ & $<0.001$ & $37.4 \pm 10.4$ & $40.6 \pm 10.8$ & NS \\
\hline Prothrombin time $(\mathrm{sec})$ & $13.6 \pm 0.9$ & $13.8 \pm 1.8$ & NS & $14.6 \pm 1.2$ & $13.2 \pm 2.0$ & NS \\
\hline Partial thromboplastin time (sec) & $45.4 \pm 12.6$ & $48.9 \pm 11.4$ & NS & $48.0 \pm 15.8$ & $49.5 \pm 7.8$ & NS \\
\hline
\end{tabular}

NS, Not significant.

Table IV. Blood products transfused in LVAS recipients according to clinical group

\begin{tabular}{|c|c|c|c|c|c|c|}
\hline Blood products (units) & Group $A$ & Group B & $\begin{array}{c}p \\
(A \cup s B)\end{array}$ & Group $B_{1}$ & Group $B_{2}$ & $\begin{array}{c}p \\
\left(B_{1} v s B_{2}\right)\end{array}$ \\
\hline Erythrocytes & $5.1 \pm 3.2$ & $14.8 \pm 12.5$ & $<0.01$ & $11.0 \pm 13.4$ & $17.4 \pm 11.8$ & NS \\
\hline Fresh-frozen plasma & $5.9 \pm 3.3$ & $14.2 \pm 14.6$ & $<0.05$ & $12.7 \pm 11.8$ & $15.2 \pm 16.8$ & NS \\
\hline Platelets & $11.1 \pm 9.6$ & $28.8 \pm 20.9$ & $<0.01$ & $24.4 \pm 14.3$ & $22.4 \pm 24.9$ & NS \\
\hline Cryoprecipitate & $9.4 \pm 8.9$ & $21.6 \pm 20.7$ & $<0.05$ & $21.4 \pm 18.6$ & $22 \pm 23.1$ & NS \\
\hline
\end{tabular}

Values represent only those amounts transfused during LVAS implantation operation and up to the point of any operation to place a right ventricular-assist device. $N S$, Not significant.

\section{Discussion}

Investigators have struggled to identify predictive factors for the development of right ventricular failure after the implantation of LVADs as a bridge to cardiac transplantation. ${ }^{3,5,9,11,12}$ In patients who preferentially receive biventricular devices, the complications from multiorgan failure, lethal arrhythmias, and low cardiac output that characterize the course of a patient in whom an inappropriate decision for univentricular support was made are avoided. ${ }^{13}$ Previous reports have examined the differences in hemodynamics and clinical variables among patients who received univentricular-assist or biventricular-assist devices; few, however, were able to prospectively follow the clinical performance of patients in whom LVADs alone were implanted who later required additional right ventricular support. This study is unique in that attempts were made to initially support all patients with the Novacor LVAS alone, with only inotropic agents and pulmonary vasodilators used to support the right ventricle. Only after it was determined that LVAS output could not be maintained above a cardiac index of $2.0 \mathrm{~L} \cdot \mathrm{min}^{-1} \cdot \mathrm{m}^{-2}$ was a right ventricularassist device inserted, so no preconceptions were made regarding the need for right ventricular support on the basis of selected preimplantation variables. The initial hemodynamic response to the
LVAS confirmed previous findings in other reports, which showed that dramatic reductions occur in the pulmonary artery wedge and mean pulmonary artery pressures, resulting in afterload reduction of the right ventricle (total pulmonary resistance) as an effect of decompression of the left ventricle by the LVAS.

There were no clear differences in hemodynamics before LVAS implantation between our clinical groups of patients. As in previous reports, rightsided and left-sided filling pressures, mean pulmonary artery pressure, heart rate, cardiac index, and right ventricular end-diastolic and end-systolic volumes did not differ between patients requiring minimal support and those requiring maximal support for the right ventricle. ${ }^{3}$ Only the preimplantation level of inotropic support differed significantly between all three groups ( $A$ vs $B ; B_{1}$ vs $B_{2}$ ), with the highest preimplantation levels being seen in those that ultimately required a right ventricular-assist device. This trend was also reflected in the preimplantation RVEF/IE ratio, which was higher in the group requiring minimal right ventricular support after LVAS. These findings are compatible with the premise that the patients who are most clinically compromised are those most likely to require biventricular support.

Certain preimplantation clinical variables did ap- 
pear different between patients who required minimal support (group A) and those who required more extensive right ventricular support (group B). Group B had more patients with mental impairment $(41 \%)$ and with active pulmonary edema $(53 \%)$, despite full medical management of heart failure. Preimplantation serum creatinine levels were higher and the mixed venous oxygen saturations were lower in this group than in group A. This is again consistent with the presumption that patients who are more clinically compromised and have more marginal end-organ function tend to require more extensive right ventricular support after LVAS implantation. A differentiating variable between those who required extensive inotropic support (group $\mathrm{B}_{1}$ ) and those who required a right ventricular-assist device (Group $\mathrm{B}_{2}$ ) was the presence of a fever within 10 days of LVAS implantation. Seven of 10 patients in group $B_{2}$ had a fever before implantation, compared with one of seven in group $B_{1}$. Fever in the absence of infection is often seen in patients in the terminal stages of end-stage heart disease and is associated with elevated levels of tumor necrosis factor, a myocardial depressant. ${ }^{14}$ The difference in early perioperative blood transfusion requirements between group $\mathrm{A}$ and $\mathrm{B}$ patients is important to note because it was caused by diffuse bleeding unrelated to obvious surgical problems before the need for a right ventricular-assist device. There was a positive correlation between the amount of blood transfused during the operative period and the total pulmonary resistance at the time of sternal closure (Pearson product-moment correlation $0.60 ; p<0.05$ ).

Analysis at autopsy or explantation failed to show major pathologic differences between hearts in the clinical groups except in thickness of the right ventricular wall. Patients who ultimately required biventricular support had a significantly thicker right ventricular free walls than did those who did not need a right ventricular-assist device. It is well recognized that right ventricular coronary blood flow depends much more on perfusion pressure than does that in the left ventricle. Presumably right ventricular myocardial perfusion may be compromised in patients with a thickened right ventricular wall. Measurements made in this fashion may have less bearing on the clinical results because pathologic examination of the heart after long periods of circulatory support may show that right ventricular wall thickness could have resolved with more extended support and that indeed hypertrophy was more likely to be seen in patients who died early and did not have the benefit of such extended support and restructuring of the cardiac muscle.

Our group of investigators and others have previously shown that the principal effect of the LVAD is reduction of left ventricular pressure and thus of left atrial pressure, with consequent reduction of pulmonary artery pressure and right ventricular afterload. ${ }^{15,16}$ Chow and Farrar ${ }^{17}$ confirmed these predictions in animal models, showing that the outcome with respect to right ventricular function was based on a complex interaction between afterload reduction and loss of right ventricular contractility related to the degree of intraventricular septal shift. ${ }^{18,19} \mathrm{We}$ have performed sophisticated analyses of right ventricular performance with the relatively load-independent parameters derived from the pressure-volume relationship obtained from transesophageal echocardiography and intraventricular pressure catheters. Contractility (end-systolic elastance) is impaired after LVAD implantation primarily as a result of loss of right ventricular function in the areas adjacent to the intraventricular septum, which shifts leftward dramatically. The beneficial effect of right ventricular afterload reduction, however, overwhelms any negative effects of reduced right ventricular contractility. ${ }^{16}$ In the face of adequately reduced right ventricular afterload, the right ventricular free wall actually appears to have improved fractional area change, despite uniformly depressed septal fractional area change. ${ }^{20}$ We therefore may conclude that right ventricular performance after LVAS implantation is highly dependent on pulmonary vascular resistance and that any factors that produce an increase in pulmonary vascular resistance may result in right ventricular failure.

One factor increasing pulmonary vascular resistance at the time of LVAD implantation may be cardiopulmonary bypass in this particularly ill group of patients. Reports have shown that cardiopulmonary bypass results in increased production of interleukins 6 and 8, with a concomitant activation of platelets, leukocytes, and complement. ${ }^{21,22}$ Inflammatory mediators rise and peak at 24 hours after bypass, along with complement C5a. Endotoxin levels have been shown to rise during cardiopulmonary bypass, ${ }^{23}$ especially during nonpulsatile perfusion. During nonpulsatile cardiopulmonary bypass, splanchnic and hepatic blood flow is decreased, further impairing the ability of the liver to clear endotoxin. ${ }^{24}$ In this series, cardiopulmonary bypass was performed with nonpulsatile perfusion by 
means of a centrifugal pump. It is hypothesized that the interruption of pulmonary flow during bypass may be responsible for alterations in vasoreactivity in the pulmonary microvasculature because histamine, which normally causes the pulmonary microvasculature to dilate, results in vasoconstriction after cardiopulmonary bypass. ${ }^{25}$ Patients with endstage heart failure who have aseptic fevers and marginal end-organ function may already have elevated levels of endotoxin and cytokines. If cardiopulmonary bypass amplifies these inflammatory mediators, including endotoxin, it is possible that a coagulopathic state may accompany the operation, resulting in a need for more blood transfusions and further compromising the pulmonary microvasculature. Increased resistance to blood flow through the pulmonary vascular bed, in combination with the negative inotropic effects produced by these mediators, could result in depressed right ventricular performance, obviating the main beneficial effect on the right ventricle of the LVAD, which is reduction of right ventricular afterload.

In conclusion, this study demonstrated that preimplantation hemodynamic values are insufficient predictors regarding which patients awaiting cardiac replacement can benefit from univentricular support alone. It suggests instead that clinical variables, which may in turn be dependent on the timing of device implantation, may play a more important role in determining the response of the right ventricle to LVAD support. In addition, perioperative factors that result in impairment of pulmonary blood flow or reduced perfusion of the right ventricle after LVAD implantation may also reduce the effectiveness of the LVAD in passive unloading of the right ventricle. Until fully elective LVAD implantation is carried out and these variables are normalized, true assessment of the outcome of univentricular support cannot be made, and the hemodynamic or biomechanical variables that predict the need for biventricular support cannot be determined.

\section{REFERENCES}

1. Kormos RL, Murali S, Dew MA, et al. Chronic mechanical circulatory support: rehabilitation, low morbidity and superior survival. Ann Thorac Surg 1994;57:51-8.

2. McCarthy PM. HeartMate implantable left ventricuiar assist device: bridge to transplantation and future applications. Ann Thorac Surg 1995;59(Suppl):S46-51.

3. McCarthy PM, Savage RM, Fraser CD, et al. Cardiopulmonary bypass, myocardial management, and support techniques: hemodynamic and physiologic changes during support with an implantable left ventricular assist device. J Thorac Cardiovasc Surg 1995;109:409-18.
4. Kormos RL, Borovetz HS, Armitage JM, Hardesty RL, Marrone GC, Griffith BP. Evolving experience with mechanical circulatory support. Ann Surg 1991;214:471-7.

5. Pennington DG, Reedy JE, Swartz MT, et al. Univentricular versus biventricular assist device support. J Heart Lung Transplant 1991;10:258-63.

6. Kormos RL, Gasior T, Antaki J, et al. Evaluation of right ventricular function during clinical left ventricular assistance. ASAIO Trans 1989;35:547-50.

7. Kormos RL, Borovetz HS, Gasior T, et al. Experience with univentricular support in mortally ill cardiac transplant candidates. Ann Thorac Surg 1990;49:261-72.

8. Kanter KR, McBride LR, Pennington DG, et al. Bridging to cardiac transplantation with pulsatile ventricular assist devices. Ann Thorac Surg 1988;46:134-40.

9. Swartz MT, Pennington DG, Ruzevich SA, et al. The incidence of isolated left ventricular failure in bridge-to-transplant candidates. ASAIO Trans 1989;35:730-3.

10. Farrar DJ, Hill JD, Thoratec Ventricular Assist Device Principal Investigators. Preoperative and postoperative comparison of patients with uni- and bi-ventricular Thoratec VAD support as a bridge to heart transplantation. $\mathbf{J}$ Heart Lung Transplant 1995;14:S68.

11. Pennington DG, McBride LR, Miller LW, Swartz MT. Eleven years' experience with the Pierce-Donachy ventricular assist device. J Heart Lung Transplant 1994;13:803-10.

12. Farrar DJ, Thoratec Ventricular Assist Device Principal Investigators. Preoperative predictors of survival in patients with Thoratec ventricular assist devices as a bridge to heart transplantation. J Heart Lung Transplant 1994;13:93-101.

13. Farrar DJ, Hill JD, LA Gray Jr, Galbraith TA, Chow E, Hershon JJ. Successful biventricular circulatory support as a bridge to cardiac transplantation during prolonged ventricular fibrillation and asystole. Circulation 1989;80(Suppl 3): III147-51.

14. Levine B, Kalman J, Mayer L, Fillit HM, Packer M. Elevated circulating levels of tumor necrosis factor in severe chronic heart failure. N Engl J Med 1990;323:236-41.

15. Brunsting LA, Salter DR, Goldstein JP, Murphy CE, AbdElfattah AS, Wechsler AS. Ventricular interaction during right ventricular assist. Trans Am Soc Artif Intern Organs 1987;33:162-8.

16. Morita S, Kormos RL, Mandarino WA, et al. Right ventricular/arterial coupling in the patient with left ventricular assistance. Circulation 1992;86(Suppl 2):II316-25.

17. Chow E, Farrar DJ. Effects of left ventricular pressure reductions on right ventricular systolic performance. Am J Physiol 1989;257:H1878-85.

18. Farrar DJ, Compton PG, Hershon JJ, Fonger JD, Hill JD. Right heart interaction with the mechanically assisted left heart. World J Surg 1985;9:89-102.

19. Farrar DJ, Compton PG, Hershon JJ, Hill JD. Right ventricular function in an operating room model of mechanical left ventricular assistance and its effects in patients with depressed left ventricular function. Circulation 1985;72:127985.

20. Kawai A, Kormos RL, Mandarino WA, et al. Differential regional function of the right ventricle during the use of a left ventricular assist device. ASAIO J 1992;38:M676-8.

21. Frering B, Philip I, Dehoux M, Rolland C, Langlois JM, Desmonts JM. Circulating cytokines in patients undergoing 
normothermic cardiopulmonary bypass. J THORAC CARDIOVASC SURG 1994;108:636-41.

22. Kalfin RE, Engelman RM, Rousou JA, et al. Induction of interleukin-8 expression during cardiopulmonary bypass. Circulation 1993;88(Pt 2):401-6.

23. Jansen NJ, van Oeveren WV, Gu YJ, van Vliet MH, Eijsman L, Wildevuur CR. Endotoxin release and tumor necrosis factor formation during cardiopulmonary bypass. Ann Thorac Surg 1992;54:744-8.

24. Watarida S, Mori A, Onoe M, et al. A clinical study on the effects of pulsatile cardiopulmonary bypass on the blood endotoxin levels. J Thorac CARdIOvasc Surg 1994;108: $620-5$.

25. Friedman M, Johnson RG, Wang SY, et al. Pulmonary microvascular responses to protamine and histamine. J THORAC CARDIOVASC SURG 1994;108:1092-9.

\section{Discussion}

Dr. J. Donald Hill (San Francisco, Calif.). This is a difficult type of clinical research because it is always done in an atmosphere of urgency and unstable patient condition. I congratulate Kormos and colleagues on their careful adherence to their protocol. This report stands alone in its pursuit of differentiating data well into the interventional period of exploring the appropriate use of LVADs. This methodology adds measurably to the credibility of the conclusions.

The first thing that struck me on reviewing the manuscript was the similarity of the results and the conclusion to those of our recent report of the 213 patients treated with a Thoratec device with respect to prediction of requirements for right ventricular support after LVAD implantation. Indeed, preoperative hemodynamic pressure data, with the exception of cardiac index, were also not helpful. Oxygen delivery to the tissues, not pressure, is the determining factor.

As in this report, we also noted elevated creatinine as a predictor, along with decreased platelet counts, use of IABP, mechanical ventilation, and emergency implantation, which occurred in $22 \%$ of the 139 patients receiving Thoratec BiVAD devices. Combining these two reports reinforces the importance of looking at the clinical condition of the patient as the differentiating factor for right ventricular support, rather than relying on hemodynamic data alone.

I made one further observation regarding this report. Implantation of a right ventricular-assist device was done some 18 to 24 hours after initial operation, with a final survival of $40 \%$. In the Thoratec data, in more than $75 \%$ of the cases the decision to add a right ventricular-assist device was made intraoperatively, presumably because of its easy availability, with a resulting posttransplantation survival rate of $83 \%$.

I point this out to emphasize the references in this report to clinical status of the patient at the time of sternal closure as an important decision point in the need for a right ventricular-assist device. I support this observation $100 \%$, and in fact learned it from Dr. Kormos several years ago.

I have several questions. One, this report is based on 40 patients who were chosen at the outset for LVAD support alone. This is a subgroup of a larger group of patients with class IV heart failure necessitating transplantation. How large was the initial group, and how often did you not even consider LVAD use alone?

Second, you emphasize the development of pulmonary vascular resistance during the operation as a prominent contributing cause of later right ventricular support requirements. Are you changing your operative procedure in any way to minimize the elevation of pulmonary vascular resistance, and if so, how?

I think this is an exceptionally good report that really gets down to the nitty-gritty of a question that has been plaguing us for 10 years. This is another step in helping us to differentiate the problem.

Dr. Kormos. I thank Dr. Hill for his comments and emphasize that the work that Hill and Farrar have done in the past 10 years has certainly motivated us to examine our patients more critically. It was the understanding of the right and left ventricular interaction that Hill and Farrar clarified that allowed us to proceed with the work that you saw today.

When we began this trial, our goal was to use the LVAD to the exclusion of all other devices. In the past year, as we have learned more about the applicability and the limitations of the LVAD, we have in fact changed our strategy. In the original series of 43 patients, however, we used the LVAD alone and only used the right ventricularassist device when it became obvious that cardiac index could not be supported by the LVAD either alone or in combination with inotropic agents.

We have now changed our strategy so that in subsets of patients who present acutely in cardiogenic shock or in the very categories that you saw here with pulmonary edema and instability of end organs, we move to a device with a potential for biventricular use, which in our institution is the Thoratec ventricular-assist device. Although we begin by using the LVAD alone, if by chest closure we see a pattern evolving with a low RVEF/IE ratio, we proceed with the addition of a Thoratec right ventricular-assist device.

With respect to changes in pulmonary vascular resistance during operation, probably no factor has changed more than our ability to control perioperative bleeding. As can be seen from our report, we believe that severe perioperative bleeding did lead to instances of right ventricular dysfunction. We now use aprotinin in selected cases, but more importantly our methods of preclotting the inflow circuits before operation has changed. Since that time, less perioperative bleeding has been encountered. Also inherent in this has been a subtle selection factor in that we now tend to choose patients for implantation who have far less deterioration in their clinical course, and this probably has something to do with intraoperative blood loss and reduces the elevation of pulmonary resistance that we saw early in the series.

Dr. Hachida (Tokyo, Japan). First, I congratulate you on your informative study and beautiful presentation. We are beginning a project with the Novacor LVAS in Japan. As you probably know, we do not have heart transplantation in our country to the bridge, so we are going to be seeing long-term LVAS use. The data you presented are crucial information for us.

I have two brief questions. First, you stated that you 
found a difference in thickness of right ventricular free wall in group $B_{2}$. Does that imply that you looked at the histology of the right ventricle in these patients? It might be one of the predictors of right ventricular dysfunction after the implantation of the LVAS, which is a contraindication for the long-term use of the Novacor device. Second, what was the duration of right ventricular failure after the LVAS implantation? When did it occur? You used inotropic agents in group $B_{1}$. When did you decide to use the right ventricularassist device after the initial implantation?

Dr. Kormos. I am not sure of the clinical significance of the finding of right ventricular hypertrophy. In many of these patients, perioperative hypotension tended to be seen very commonly, and as you know the right ventricle is so dependent on coronary perfusion pressure that it is possible that with hypotension a hypertrophic right ventricle performed less well. Other biopsy findings that we looked at included the percentage of fibrosis and other pathologic findings, although we did not find any significant differences between the groups with respect to those other pathologic findings.

With respect to when right ventricular failure developed, all of the patients reported on here had right ventricular-assist devices implanted if they needed them somewhere between 8 to 48 hours after LVAS implantation. In retrospect, we probably could have made the diagnosis of right ventricular failure at the time of chest closure or at the time the patient left the operating room, but for study reasons we persisted with inotropic agents. I believe now that, as in Dr. Hill's findings, a more rapid institution of biventricular support probably certainly would have led to a higher survival and a higher transplantation rate for patients with severe biventricular failure.

\section{1-800-55-MOSBY}

This number links you to the full text of articles published in over 25,000 journals, including all Mosby journals. MOSBY Document Express $^{\circledR}$, a rapid response information retrieval service, provides quick turnaround, 24-hour availability, and speedy delivery methods. For inquiries and pricing information, call our toll-free, 24-hour order line: 1-800-55-MOSBY; outside the United States: 415-259-5046; fax: 415-259-5019; E-mail: mosbyexp@class.org.

MOSBY Document Express ${ }^{\circledast}$ is offered in cooperation with Dynamic Information Corp. 